\title{
Sirt3 protects in vitro-fertilized mouse preimplantation embryos against oxidative stress-induced p53-mediated developmental arrest
}

\author{
Yumiko Kawamura, ${ }^{1}$ Yasunobu Uchijima, ${ }^{1}$ Nanao Horike, ${ }^{1}$ Kazuo Tonami, ${ }^{1}$ Koichi Nishiyama, ${ }^{1}$ \\ Tomokazu Amano, ${ }^{1}$ Tomoichiro Asano, ${ }^{2}$ Yukiko Kurihara, ${ }^{1}$ and Hiroki Kurihara ${ }^{1}$
}

1Department of Physiological Chemistry and Metabolism, Graduate School of Medicine, University of Tokyo, Tokyo, Japan. 2Department of Biomedical Chemistry, Hiroshima University Graduate School of Biomedical Sciences, Hiroshima, Japan.

\begin{abstract}
Sirtuins are a phylogenetically conserved $\mathrm{NAD}^{+}$-dependent protein deacetylase/ADP-ribosyltransferase family implicated in diverse biological processes. Several family members localize to mitochondria, the function of which is thought to determine the developmental potential of preimplantation embryos. We have therefore characterized the role of sirtuins in mouse preimplantation development under in vitro culture conditions. All sirtuin members were expressed in eggs, and their expression gradually decreased until the blastocyst stage. Treatment with sirtuin inhibitors resulted in increased intracellular ROS levels and decreased blastocyst formation. These effects were recapitulated by siRNA-induced knockdown of Sirt3, which is involved in mitochondrial energy metabolism, and in Sirt $3^{-/}$embryos. The antioxidant $N$-acetyl- $L$-cysteine and lowoxygen conditions rescued these adverse effects. When Sirt3-knockdown embryos were transferred to pseudopregnant mice after long-term culture, implantation and fetal growth rates were decreased, indicating that Sirt3-knockdown embryos were sensitive to in vitro conditions and that the effect was long lasting. Further experiments revealed that maternally derived Sirt3 was critical. Sirt3 inactivation increased mitochondrial ROS production, leading to $\mathrm{p} 53$ upregulation and changes in downstream gene expression. The inactivation of p53 improved the developmental outcome of Sirt3-knockdown embryos, indicating that the ROS-p53 pathway was responsible for the developmental defects. These results indicate that Sirt3 plays a protective role in preimplantation embryos against stress conditions during in vitro fertilization and culture.
\end{abstract}

\section{Introduction}

Infertility, defined as failure to conceive after 2 years of unprotected intercourse, is a major social and personal problem affecting approximately $10 \%$ of couples worldwide. Assisted reproductive technologies, such as in vitro fertilization (IVF), are widely used for the treatment of infertility in clinical practice, but many issues impeding therapeutic efficacy remain to be solved (1-3). In general, human reproduction is inefficient, with more than $60 \%$ of fertilized embryos unable to survive to delivery, mainly because of early developmental failure before and during implantation $(4,5)$. Although various intrinsic and environmental factors have been proposed to affect early embryogenesis, the mechanisms determining developmental potential remain largely unveiled.

It has recently been postulated that mitochondrial function is a possible determinant of developmental potential in preimplantation embryos $(6,7)$. Although mitochondria within eggs and preimplantation embryos are morphologically premature and their oxygen consumption is relatively low, blockade of oxidative phosphorylation and perturbation of mitochondrial membrane integrity exerts a detrimental effect on preimplantation development (8-10). Furthermore, ATP content within individual eggs is correlated with reduced developmental competence and postimplantation outcomes (11). In addition to ATP synthesis through oxidative phos-

Conflict of interest: The authors have declared that no conflict of interest exists. Citation for this article: J Clin Invest. 2010;120(8):2817-2828. doi:10.1172/JCI42020. phorylation, mitochondria play diverse roles in signal transduction, calcium homeostasis, and oxygen metabolism. In particular, ROS generated in mitochondrial electron transport chain, together with cytoplasmic $\mathrm{NAD}(\mathrm{P}) \mathrm{H}$ oxidase and xanthine oxidase, has been implicated in various biological processes (12-14). Oxidative stress caused by excessive production of ROS and/or impaired antioxidant defense mechanisms has been shown to be detrimental to germ cells and fertilized eggs, resulting in the impairment of early embryonic development (15-17). Involvement of mitochondrial dysfunction in this process has also been reported (18).

Recently, the sirtuins, deacetylase/ADP-ribosyltransferase proteins with homology to the yeast silent information regulator 2 (Sir2), have emerged as a key mediator between energy metabolism and cellular signaling in various species (19-21). The lysine deacetylation activity of sirtuins is characteristically upregulated by $\mathrm{NAD}^{+}$and downregulated by NADH and nicotinamide, a product of the deacetylation reaction; sirtuins are thereby postulated to link the cellular energy state to biochemical signals by sensing $\mathrm{NAD}^{+} / \mathrm{NADH}$ levels $(22,23)$. In mammals, 7 proteins (Sirt1-Sirt7) are known to constitute the sirtuin family and are implicated in many physiological events, such as aging, cell metabolism, apoptosis, and cell cycle regulation (19-21). Among the sirtuin members, Sirt3, Sirt4, and Sirt5 are characterized by their localization to the mitochondria (24-27). Previous studies have revealed that Sirt3 is involved in the regulation of mitochondrial proteins such as acetylCoA synthase 2 (AceCS2) through its deacetylation activity $(28,29)$. 
A

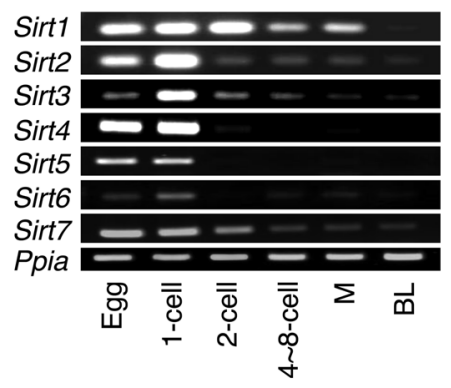

B

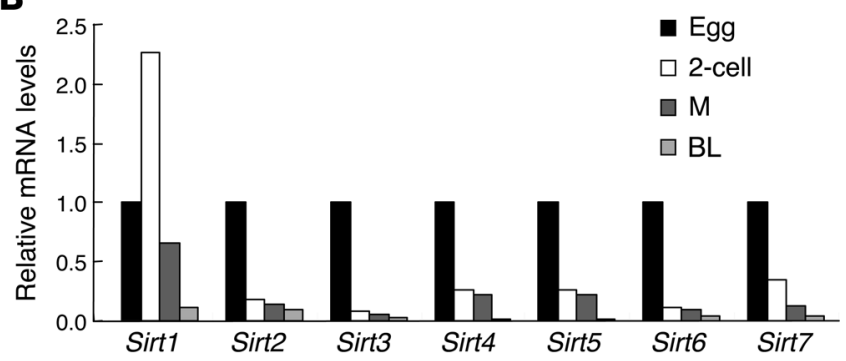

In addition, gene-knockout experiments have elucidated the role of Sirt3 in the regulation of mitochondrial activity and basal ATP synthesis (30). However, Sirt3 $3^{-/}$mice demonstrate no obvious phenotype in spite of a 50\% reduction in ATP levels, which indicates that the basal metabolic state could stand this reduction, but phenotype might become apparent under certain stress conditions $(30,31)$.

In the present study, we investigated whether sirtuin might serve as a contributing factor to the developmental potential of preimplantation embryos. We demonstrated that Sirt3 played a protective role in mouse preimplantation embryos under in vitro culture conditions. Whereas Sirt $3^{-/-}$mice were fertile, IVF and in vitro-cultured Sirt3-/- or Sirt3 siRNA-induced knockdown embryos were susceptible to developmental defects. Our results further indicated the involvement of tumor suppressor p53 induction, possibly triggered by mitochondrial ROS, in Sirt3 deficiency-induced developmental arrest. These findings may implicate Sirt3 activity in successful IVF outcome as a regulator of mitochondrial function.

\section{Results}

Sirtuins are expressed in mouse eggs and preimplantation embryos. To investigate the possible involvement of sirtuins in preimplantation development, we first examined the expression of Sirt1-Sirt7 genes in eggs and early embryos using specific primers (Supplemental Table 1; supplemental material available online with this article; doi:10.1172/JCI42020DS1). In eggs and early embryos, expression of all the sirtuin members was detected by RT-PCR (Figure 1). After the first cleavage, Sirt1-Sirt7 expression was downregulated with distinct time courses (Figure 1).

Sirtuin inbibitors cause developmental defects and increased mitochondrial ROS generation in preimplantation embryos. We next examined whether blockade of sirtuin activities affects preimplantation development. Nicotinamide, a product of the sirtuin deacetylation reaction and an inhibitor of sirtuin activity, has been reported to suppress blastocyst formation and subsequent postimplantation development (32). Consistently, nicotinamide, but not nicotinic acid, inhibited preimplantation development after IVF (Figure 2A) as early as the second cleavage stage (Supple-

\section{Figure 1}

Sirtuin gene expression in mouse eggs and preimplantation embryos. (A) Conventional RT-PCR analysis. Eggs and preimplantation embryos were collected for RNA sampling from the oviducts or uteri at the appropriate time for each stage as follows: egg, 1-cell, 2-cell, approximately 4- to 8-cell, morula (M), and blastocyst (BL). Ppia expression served as an internal control. (B) Relative quantification of sirtuin mRNA levels by real-time RT-PCR. mental Figure 1). In addition, 2 other sirtuin deacetylase inhibitors, sirtinol and $\mathrm{N}$-(2-aminophenyl)- $\mathrm{N}^{\prime}$-phenyloctanediamide (BML-210), also inhibited development after IVF, with stage profiles similar to that of nicotinamide treatment (Figure 2B and Supplemental Figure 2).

In another series of experiments, we detected an increase in the fluorescence intensity emitted by 5-(and-6)-chloromethyl-2', $7^{\prime}$ dichlorodihydrofluorescein diacetate (CM- $\left.\mathrm{H}_{2} \mathrm{DCFDA}\right)$ fluorescent dye in sirtinol-treated embryos. This increase in fluorescent signals was abolished by treatment with the antioxidant $N$-acetyl- $L$-cysteine (NAC; Figure 2, C and E) and the mitochondrial complex III inhibitor stigmatellin (Figure 2, D and F), which also inhibits complex I at higher concentrations (33). Our observations indicate that intracellular ROS levels were increased by sirtinol, and excessive ROS generation by mitochondria appeared to be responsible for this phenomenon. These findings suggest the possible involvement of sirtuin activity in normal progression of preimplantation development and control of intracellular ROS levels.

Sirtuins are upregulated by oxidative stress. During fertilization and preimplantation development in vitro, oxidative stress imposed by excessive intracellular ROS levels is supposed to cause a harmful effect on gametes and embryos (15-17). Therefore, we speculated that the expression of sirtuins might be upregulated by oxidative stress to provide protective feedback in early embryos. To test this possibility, we incubated 4-cell embryos with media containing $\mathrm{H}_{2} \mathrm{O}_{2}$ and examined changes in sirtuin mRNA levels by RT-PCR. Exposure to $20 \mu \mathrm{M} \mathrm{H}_{2} \mathrm{O}_{2}$ for 6 hours resulted in increased Sirt3 expression in embryos (Figure 3A). $\mathrm{H}_{2} \mathrm{O}_{2}$-induced increases in Sirt3 protein levels were confirmed in embryos at the same stage (Figure 3B) and in NIH 3T3 cells (Figure 3C). Because Sirt3 is a mitochondrial deacetylase involved in the regulation of mitochondrial electron transport, and because mitochondria are the major source of ROS and a determinant of developmental competence of preimplantation embryos $(6,9,34)$, we therefore focused on Sirt3 in subsequent experiments.

Sirt3 is localized to mitochondria in eggs and preimplantation embryos. Sirt3 has been described as a mitochondrial sirtuin, but recent studies have reported that it is also localized to the nucleus in 
A

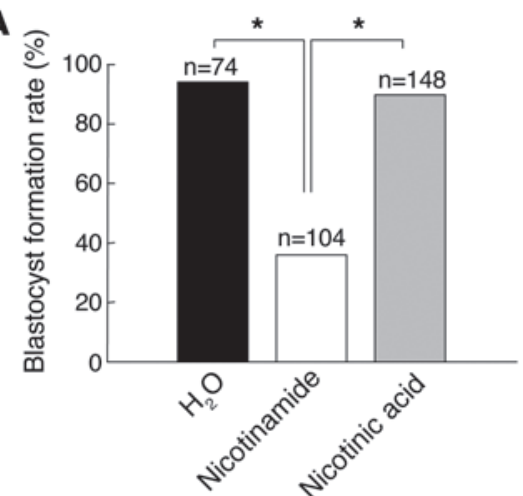

C

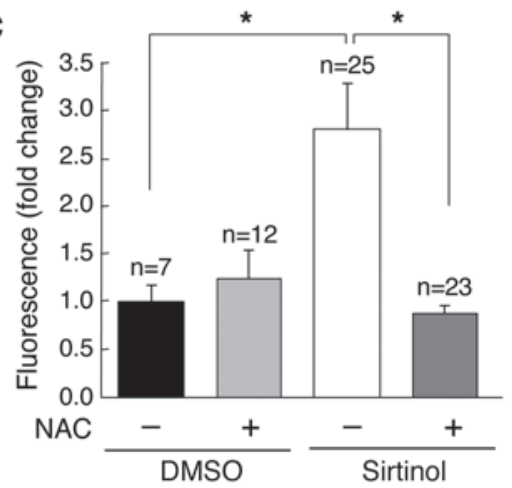

E

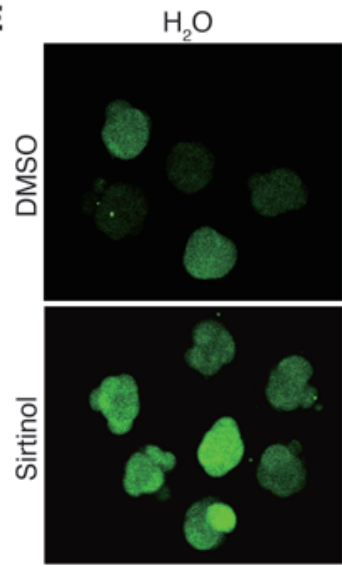

NAC

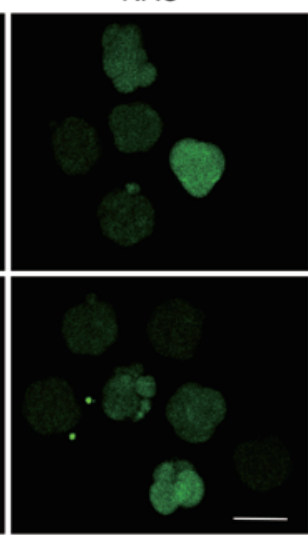

B

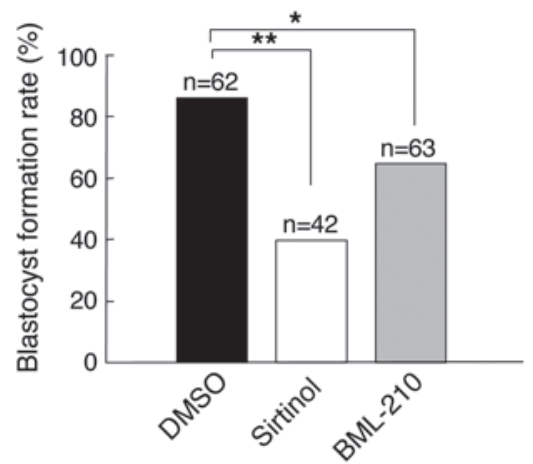

D

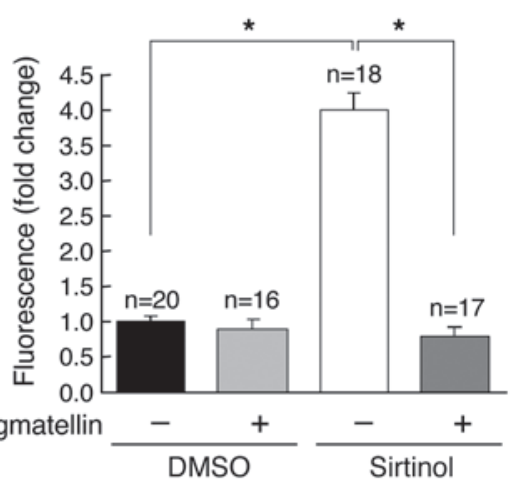

F
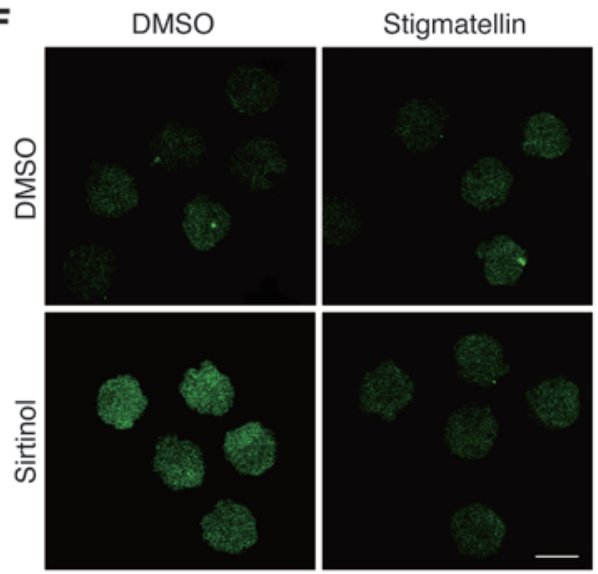

Figure 2

Sirtuin inhibitors cause decreased blastocyst formation and increased mitochondrial ROS generation in preimplantation embryos. (A and B) The sirtuin inhibitors nicotinamide, sirtinol, and BML-210 caused developmental arrest. Embryos were treated with inhibitors during IVF and in vitro culture, and the blastocyst formation rate was calculated by dividing the number of blastocysts by the number of 2-cell embryos. Nicotinic acid, a nicotinamide derivative, had no effect on developmental outcome. $\mathrm{H}_{2} \mathrm{O}$ and DMSO (final concentration, $0.2 \%$ ) served as control for each experiment. Data are derived from 7 independent experiments. Statistical assessments were performed by applying Ryan's multiple-comparison test. ${ }^{\star} P<0.05 ;{ }^{* *} P<0.001$. (C and D) Sirtinol increased intracellular ROS levels, as estimated by $\mathrm{CM}-\mathrm{H}_{2} \mathrm{DCFDA}$ fluorescence intensity. This increase was blocked by NAC (C) and stigmatellin (D). Embryos were treated with the indicated agents for 72 hours. Quantitative data of fluorescence intensity, obtained using ImageJ, were standardized by dividing each value by the average value of the control group in each experiment. Data are derived from 3 independent experiments. Statistical assessments were performed by applying Games-Howell test. ${ }^{*} P<0.05$. (E and $\left.\mathbf{F}\right)$ Representative images of $C M-\mathrm{H}_{2}$ DCFDA fluorescence in embryos analyzed in C and D, respectively. Scale bars: $100 \mu \mathrm{m}$.

some cell types (25). To examine whether Sirt3 localizes to mitochondria in preimplantation embryos, we injected in vitro-transcribed Sirt3-EGFP mRNA into fertilized eggs and observed EGFP fluorescence at the 2-cell stage. The distribution pattern of Sirt3-EGFP fusion protein was granular within the cytoplasm (Figure 4A). Costaining with MitoTracker showed colocalization of Sirt3-EGFP to mitochondria, as expected (Figure 4, B and C). In contrast, Sirt1-EGFP (Figure 4, D-F) and Sirt2-EGFP (Figure $4, \mathrm{G}-\mathrm{I}$ ) were localized to the nucleus and cytoplasm, respectively. EGFP alone was distributed throughout the cell (Figure 4J). These results indicate that Sirt 3 is likely to be localized to mitochondria in mouse preimplantation embryos. 

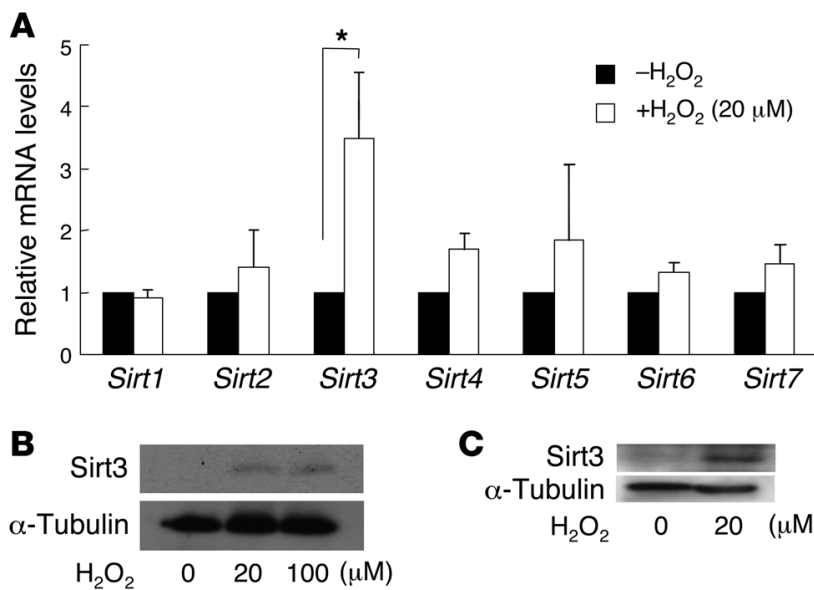

RNAi-mediated Sirt3 knockdown increases mitochondrial ROS generation, causing developmental arrest in preimplantation embryos. To investigate the role of Sirt3 in preimplantation development, we selectively knocked down the expression of Sirt3 by injecting Sirt3-targeted siRNA into fertilized eggs. We designed 2 stealth siRNAs to target 2 different regions of the Sirt 3 transcript (siRNAs 1 and 2; see Methods and Supplemental Table 2). Both siRNAs downregulated Sirt 3 mRNA and protein in NIH 3 T3 cells (Supplemental Figure 3, A and B), and the efficacy was confirmed in 8-cell to morula stage embryos (Supplemental Figure 3, C and D). The expression of other sirtuin family members was not affected by Sirt3 siRNA (Supplemental Figure 3E).

We next examined whether Sirt3 knockdown increases intracellular ROS levels by using $\mathrm{CM}-\mathrm{H}_{2}$ DCFDA fluorescence dye. In Sirt3 siRNA-injected embryos, CM- $\mathrm{H}_{2}$ DCFDA fluorescence was significantly higher than that in control embryos (Figure 5, A-D). This increase in fluorescence intensity was abolished by NAC (Figure 5, A and C) and stigmatellin (Figure 5, B and D), as in sirtinoltreated embryos. Apocynin, a selective inhibitor of $\mathrm{NAD}(\mathrm{P}) \mathrm{H}$ oxidase, also partially suppressed this increase, but the effect was less than that of stigmatellin (Figure 5, B and D). These results suggest that Sirt3 inactivation may cause intracellular ROS production, mainly of mitochondrial origin.

Embryos injected with either of the stealth Sirt3 siRNAs exhibited a significant decrease in blastocyst formation rate, whereas fertilization rate, estimated by the formation of 2-cell embryos, was not affected (Figure 6A and Supplemental Figure 4). This decrease in blastocyst formation was suppressed by NAC (Figure $6 \mathrm{~B})$, which indicates that increased ROS is possibly responsible for developmental arrest in Sirt3-knockdown embryos.

Sirt3 knockdown-induced developmental defects are abolished in lowoxygen conditions. The results of our Sirt3-knockdown experiments suggested that in vitro culture conditions are stressing the embryos to induce increased ROS and developmental defects in the absence of sufficient Sirt3 activity. To test this possibility, we performed the same experiments under low-oxygen $\left(5 \% \mathrm{O}_{2}\right)$ conditions. As expected, Sirt3 knockdown did not affect 2-cell and blastocyst formation rates in low-oxygen conditions (Figure 7A). Sirt3 knockdown-induced increase in intracellular ROS levels was also abolished in low-oxygen conditions (Figure 7, B and C). These results indicate that Sirt3 may play a protective role for preimplantation embryos within a stressful environment.

\section{Figure 3}

Oxidative stress upregulates the expression of sirtuins. (A) Quantitative real-time RT-PCR analysis of sirtuin gene expression after $\mathrm{H}_{2} \mathrm{O}_{2}$ treatment $(20 \mu \mathrm{M}, 6$ hours) in preimplantation embryos. Sirt3 mRNA levels were significantly increased in 4-cell embryos after exposure to $\mathrm{H}_{2} \mathrm{O}_{2}$. Data are derived from 3 independent experiments. Statistical assessments were performed by applying Mann-Whitney $U$ test. ${ }^{\star} P<0.05$. (B and $\mathbf{C}$ ) Western blotting analysis showing Sirt3 protein upregulation in 4-cell embryos (B) and NIH 3T3 cells (C) after $\mathrm{H}_{2} \mathrm{O}_{2}$ treatment (B, 20 and $100 \mu \mathrm{M}, 18$ hours; $\mathbf{C}, 20 \mu \mathrm{M}, 24$ hours) detected by Western blot. Blotting for $\alpha$-tubulin served as an internal control.

Sirt3-null eggs are defective in developmental competence after IVF and parthenogenesis. To confirm the results from siRNA-knockdown experiments, we performed similar experiments using embryos genetically deficient in Sirt3. Gametes with the Sirt $3^{+}$or Sirt $3^{-}$allele were prepared from $\mathrm{Sirt}_{3^{+/+}}$and Sirt $3^{-/-}$littermates, respectively, and were subjected to IVF. Unlike Sirt3 siRNA-injected embryos, fertilization rate significantly decreased in embryos derived from Sirt3- eggs, regardless of paternal genotype (Table 1). This difference may be explained by the lack of maternally derived Sirt3 storage in Sirt3- eggs. In addition, blastocyst formation rate under in vitro culture conditions was also significantly decreased in embryos derived from Sirt3- eggs (Table 2). These results indicate that Sirt3 of maternal origin plays an important role in fertilization and preimplantation development under in vitro conditions.

The role of maternally derived Sirt 3 in preimplantation development was further confirmed by examining development of parthenogenetic embryos with different Sirt3 levels. Eggs injected with Sirt3 and control siRNA developed to the 2-cell stage after parthenogenetic activation by $\mathrm{SrCl}_{2}\left(\mathrm{Sr}^{2+}\right)$ at comparable rates (Figure $\left.8 \mathrm{~A}\right)$. Conversely, blastocyst formation was significantly compromised in parthenogenetic embryos injected with Sirt3 siRNA (Figure 8A). Similar results were obtained using eggs with the Sirt $3^{+}$or Sirt $3^{-}$alleles. Parthenogenetically activated embryos deficient in Sirt3 developed well to the 2-cell stage, but their blastocyst formation rates decreased (Figure $8 \mathrm{~B})$. These results confirmed the importance of maternally derived Sirt3 for preimplantation developmental competence.

Long-term in vitro culture leads to defective postimplantation development in Sirt3-knockdown embryos. In contrast to our present finding, Sirt $3^{-/-}$mice have previously been shown to be healthy under basal conditions and fertile (31). However, another group has reported that cells from Sirt3 $3^{-/}$mice exhibit reduced basal ATP levels and increased acetylation of mitochondrial proteins (30). Therefore, we speculated that Sirt3 might not be required for development under natural optimal conditions, but functions under stressful conditions, such as in vitro culture. To explore this possibility, we transferred siRNA-injected embryos to pseudopregnant mice after short- or long-term in vitro culture and assessed the implantation rate and postimplantation survival of Sirt3-knockdown embryos. When embryos were transferred at the 2-cell stage, the rates of implantation and full-term survival were not significantly affected by Sirt3 knockdown (Figure 9, A and B). Conversely, implantation rate and full-term survival were significantly decreased in Sirt3 

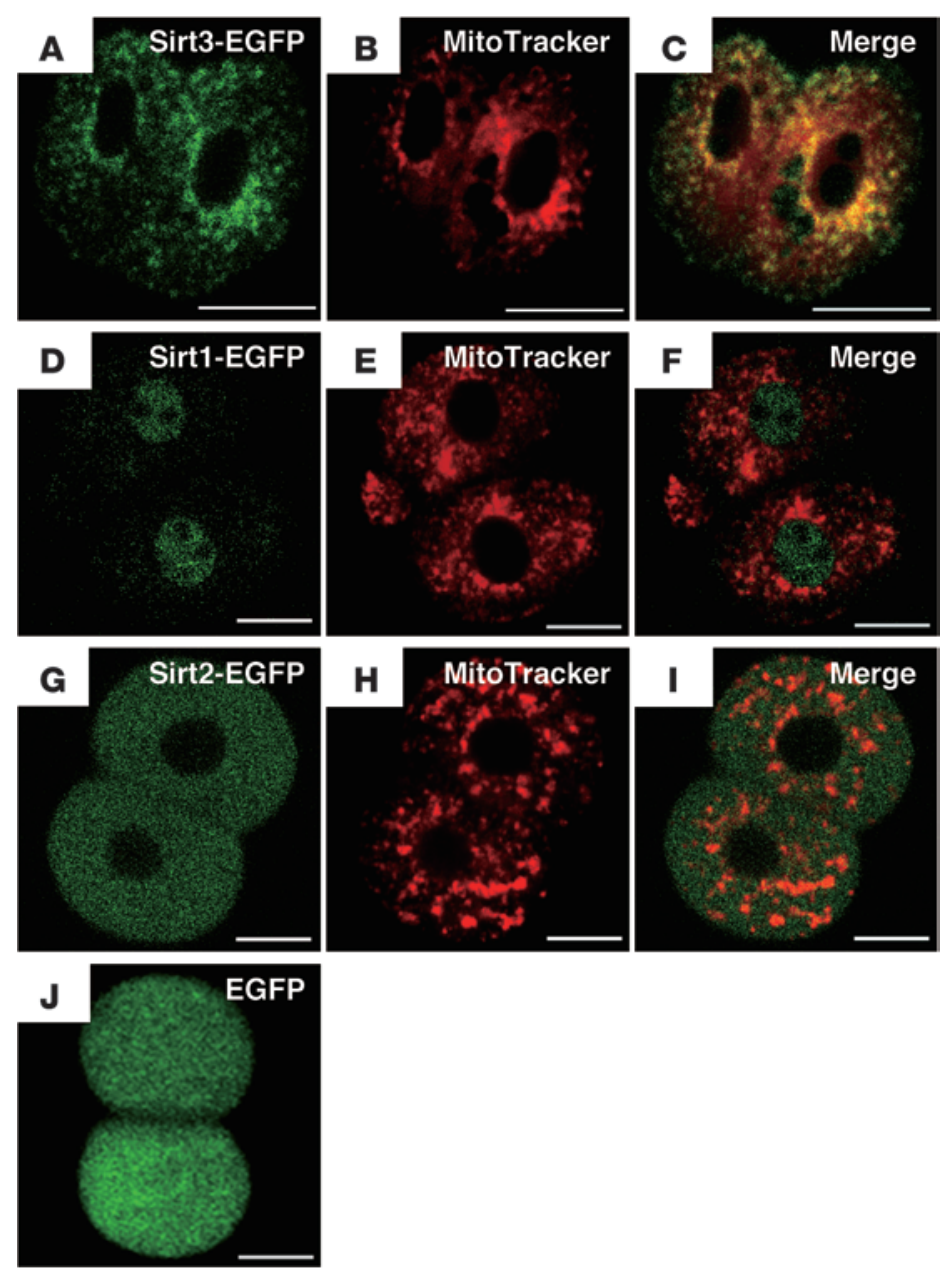

Figure 4

Confocal imaging analysis of the localization of sirtuin-EGFP fusion proteins in preimplantation embryos. Intracellular injection of mRNA coding Sirt3EGFP (A-C), Sirt1-EGFP (D-F), Sirt2-EGFP (G-I), or EGFP alone (J) was performed at the pronuclear stage, and the EGFP signals $(\mathbf{A}, \mathbf{D}, \mathbf{G}$, and $\mathbf{J}$; green) were observed at the 2-cell stage. (B, E, and $\mathbf{H}$ ) Mitochondria were stained by MitoTracker (red) just 15 minutes before observation. (C, F, and I) Colocalization of Sirt3-EGFP signals to MitoTracker staining confirmed mitochondrial localization of Sirt3. Scale bars: $20 \mu \mathrm{m}$.

siRNA-treated embryos when transferred at the morula/blastocyst stage, 72-96 hours after siRNA injection (Figure 9, A and B). These results suggest that Sirt3 is required for embryos to establish a successful pregnancy after long-term in vitro culture.

p53 is involved in developmental arrest due to Sirt 3 deficiency. Increased intracellular ROS is known to trigger the activation of the tumor suppressor $\mathrm{p} 53$, leading to cell cycle arrest and apoptosis in various cell types $(35,36)$. Many functions of p53 are attributed to its transcriptional activity on various gene promoters, including that of the cyclin-dependent kinase inhibitor p21 $(37,38)$. Recently, p53 has been reported to induce the differentiation of ES cells by suppressing the expression of Nanog homeobox (Nanog), a transcription factor required for ES cell self-renewal, after DNA damage $(39,40)$. To investigate whether p53 is involved in developmental arrest in Sirt3-knockdown or knockout embryos, we examined the levels of p53 and its target gene expression. In Sirt3 $3^{-/}$early embryos, $p 53$ and p21 expression was upregulated, whereas Nanog expression was downregulated (Figure 10A and Supplemental Figure 5A). Similar expression patterns were recapitulated by treatment with $\mathrm{H}_{2} \mathrm{O}_{2}$ in wild-type embryos (Figure 10A and Supplemental Figure 5A). Involvement of p53 was further investigated using siRNA-mediated knockdown (Supplemental Table 2). Injection of both control and Sirt3 siRNA appeared to increase $p 53$ mRNA to a similar level, possibly as a result of stimuli caused by the microinjection procedure (Figure 10B and Supplemental Figure 5B). Treatment with NAC decreased p53 mRNA levels only in control siRNA-injected embryos, revealing a difference in $p 53$ expression between control and Sirt3 siRNA embryos similar to that between wild-type and Sirt3 $3^{-/}$embryos (Figure 10B and Supplemental Figure 5B). In contrast, p53 protein levels were increased in Sirt3-knockdown embryos without NAC treatment (Figure 10C), which indicates that p53 protein is stabilized in Sirt3-knockdown embryos. Accordingly, $p 21$ expression was upregulated and Nanog was downregulated, as observed in Sirt $3^{-1-}$ embryos (Figure 10B and Supplemental Figure 5B). These effects were suppressed by NAC (Figure 10B and Supplemental Figure $5 \mathrm{~B}$ ), which indicates that p53 is stabilized and activated by ROS in Sirt3-knockdown embryos.

Finally, we examined the effect of siRNA-mediated p53 knockdown on development of Sirt3-knockdown embryos to test whether the increased p53 is involved in developmental arrest caused by Sirt 3 deficiency. Coinjection with p53 siRNA effectively downregulated p53 and p21 expression and upregulated Nanog expression in Sirt3 siRNA-injected embryos (Figure 10D and Supplemental Figure 5C). Furthermore, the rate of blastocyst formation was significantly improved in Sirt3-knockdown embryos by coinjection with p53 siRNA (Figure 10E). These results indicate that ROS-induced p53 activation contributes to developmental arrest in Sirt3-knockdown embryos under in vitro culture conditions.

\section{Discussion}

In the present study, we demonstrated the involvement of sirtuins, especially Sirt3, in mouse preimplantation development. Early expression patterns and developmental arrest by sirtuin inhibitors suggest the involvement of this protein family in preimplantation development. Among them, Sirt3, predominantly of maternal origin, was shown to be required for in vitro preimplantation development by experiments using siRNA-induced knockdown and gene-knockout mice. In apparent contrast to previous studies reporting that Sirt3-deficient mice demonstrate no obvious phenotype in fertility, although they show mitochondrial protein hyperacetylation and reduction in basal ATP levels $(30,31)$, our present findings indicate that the Sirt3-null phenotype becomes evident when embryos are exposed to stress conditions. In IVF and in vitro-cultured embryos, Sirt3 inactivation caused increased ROS production, which led to p53 activation and subsequent developmental arrest. Collectively, our present results identified Sirt3 as one of the key factors determining the developmental outcome of IVF embryos, at least in mice.

Maternal and zygotic expression of sirtuins in eggs and preimplantation embryos. All sirtuins were expressed in eggs, and the expression levels gradually decreased during preimplantation development, which indicated that they were transcribed and stored 
A

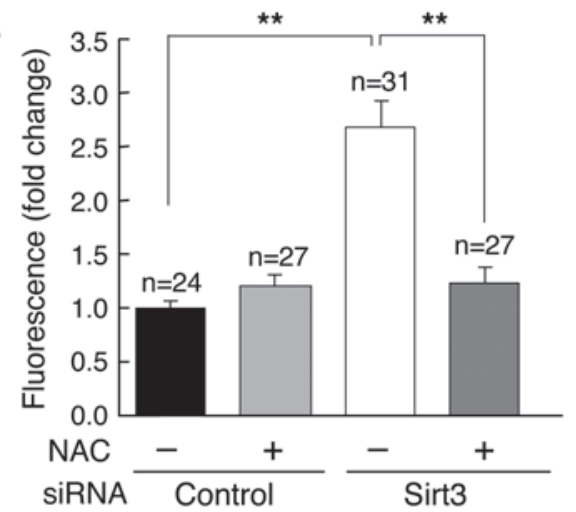

C

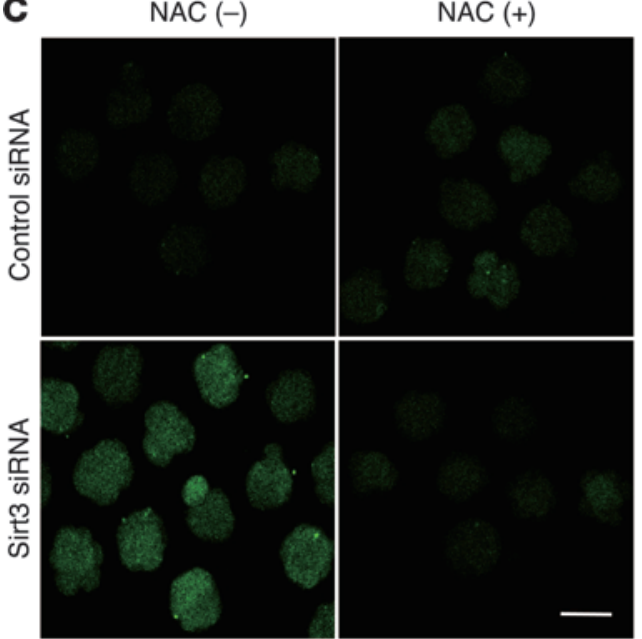

B

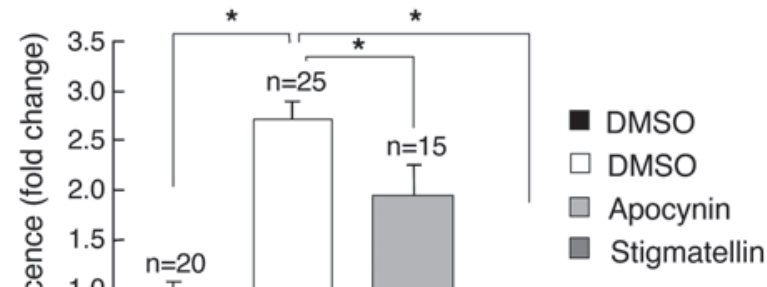

D
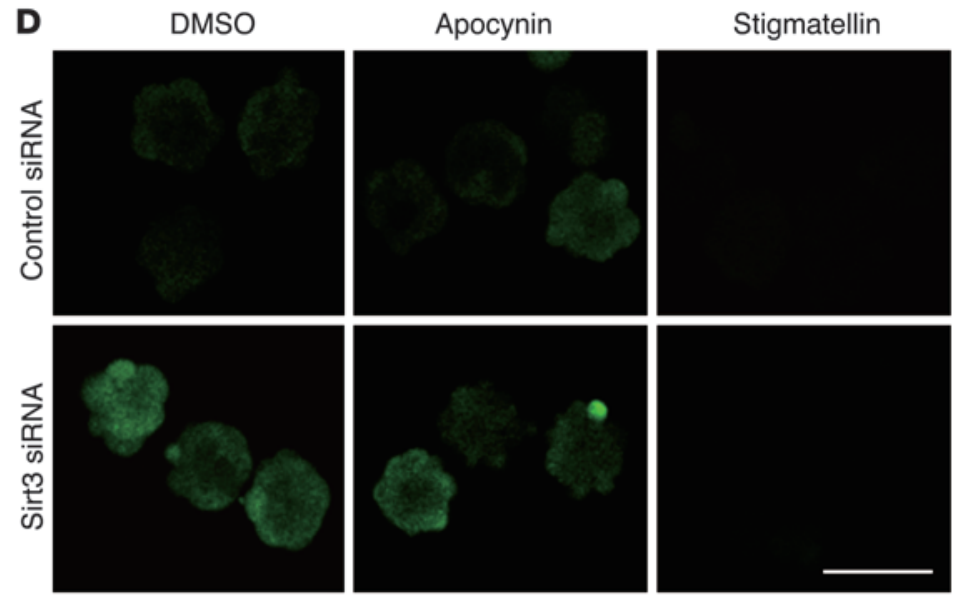

\section{Figure 5}

Sirt3 knockdown increases intracellular ROS, mainly of mitochondrial origin, in preimplantation embryos. (A and B) Injection of Sirt3 siRNA increased intracellular ROS levels, as estimated by $\mathrm{CM}-\mathrm{H}_{2}$ DCFDA fluorescence intensity. This increase was abolished by NAC (A) and stigmatellin (B), but was only partially decreased by apocynin (B). Embryos were injected with control or Sirt3 siRNA at the pronuclear stage and were cultured with or without NAC for 72 hours. To identify the major origin of increased intracellular ROS, embryos were treated with apocynin or stigmatellin for 30 minutes before $\mathrm{CM}-\mathrm{H}_{2}$ DCFDA staining. Quantitative data of fluorescence intensity, obtained using ImageJ, were standardized by dividing each value by the average value of the control group in each experiment. Data are derived from 3 (A) or 4 (B) independent experiments. Statistical assessments were performed by applying Games-Howell test. ${ }^{\star} P<0.05 ;{ }^{* \star} P<0.001$. (C and $\left.\mathbf{D}\right)$ Representative images of $\mathrm{CM}-\mathrm{H}_{2}$ DCFDA fluorescence in embryos analyzed in $\mathbf{A}$ and $\mathbf{B}$, respectively. Scale bars: $100 \mu \mathrm{m}$.

during the process of oogenesis but their zygotic expression was relatively low or undetectable. In contrast, the expression of some sirtuins, including Sirt3, was upregulated by oxidative stress in zygotes. This is in agreement with previous reports that Sirt3 is a stress-responsive deacetylase in other cell types (41). Although the mechanism of the stress-induced upregulation is unclear, this finding suggests that zygotic expression of some sirtuin genes can be induced in stress conditions. However, the developmental competence of Sirt3-null eggs after IVF was reduced regardless of the sperm genotype. Thus, we conclude that maternally derived Sirt3 is critical for normal preimplantation development after IVF, whereas zygotically activated Sirt 3 is not sufficient to compensate the loss of maternal Sirt3.

Regulation of mitochondrial ROS generation by Sirt3. Sirt3 is regarded as the major mitochondrial deacetylase involved in the regulation of energy metabolism (19-21). Mitochondria are not only the site of oxidative phosphorylation and energy production, but also a major source of ROS. Excessive ROS generation caused by mitochondrial dysfunction leads to oxidative stress, which is implicated in pathophysiological processes such as genomic instability and aging (42). In preimplantation development, mitochondrial dysfunction and subsequent ROS-induced oxidative stress have been shown to adversely affect developmental outcome $(9,18)$, indicating the importance of protective mechanisms against excessive ROS generation. The present study has shown that Sirt3-knockdown preimplantation embryos exhibited an increase in intracellular ROS, mainly of mitochondrial origin. This finding is complementary to previous studies demonstrating that overexpression of wild-type Sirt3 decreased intracellular ROS, whereas overexpression deacetylase-defective mutant Sirt3 increased it $(27,43)$. In addition, decreased blastocyst formation rates were improved by the antioxidant NAC, which indicated that the increase in ROS was responsible for developmental arrest. Furthermore, lowering oxygen concentration in culture 
A

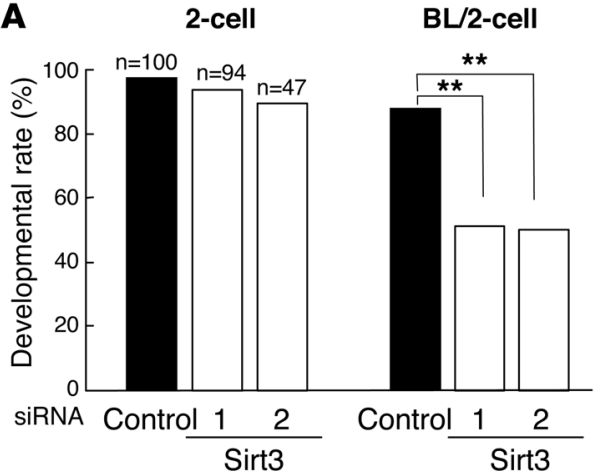

B

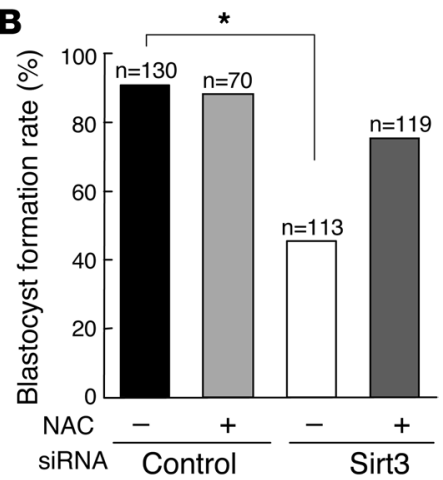

\section{Figure 6}

RNAi-mediated Sirt3 knockdown adversely affects preimplantation developmental outcomes. (A) Intracytoplasmic injection of both stealth Sirt3 siRNAs led to decreased blastocyst formation rate in preimplantation embryos. Data are derived from 4 independent experiments. (B) Sirt3 siRNA-induced decrease in blastocyst formation rate was suppressed by treatment with NAC. Data are derived from 6 independent experiments. Statistical assessments were performed by applying Ryan's multiple-comparison test. ${ }^{*} P<0.05$; ${ }^{* *} P<0.001$.

conditions abolished Sirt3 knockdown-induced developmental defects. These findings suggest that Sirt3 plays a protective role against oxidative stress-induced developmental arrest in IVF and in vitro-cultured preimplantation embryos.

Within mitochondria, the primary sites of ROS generation are complexes I and III of the electron transport chain (12-14). Accumulation of electron donors as a result of reduced electron flow and increased oxygen concentration is supposed to cause excessive generation of superoxide as the primary product (44). Enhanced ROS production induced by Sirt3 inactivation was blocked by stigmatellin, which inhibits electron flux at complexes I and III (33). In this vein, it is noteworthy that Sirt3 deficiency led to inhibition of complex I activity, possibly through hyperacetylation of multiple components of complex I, such as NDUFA9 (30). Thus, alterations in electron transfer activity of complex I may be responsible for increased mitochondrial ROS generation in Sirt3-knockdown embryos. In addition, Sirt 3 expression was upregulated by $\mathrm{H}_{2} \mathrm{O}_{2}$ in preimplantation embryos. This may serve as a feedback mechanism to regulate intracellular ROS levels.

Recent studies have shown that Sirt3 interacts with Foxo3a, a forkhead box-containing transcription factor, to upregulate the expression of manganese superoxide dismutase and catalase, thereby decreasing intracellular ROS levels in cardiomyocytes and suppressing cardiac hypertrophy (45). Upon deacetylation by Sirt3, Foxo3a translocates to the nucleus to transactivate the antioxidant genes (45). However, our preliminary experiments did not detect any differences in Foxo3a localization or the expression of catalase and Mn-superoxide dismutase genes, downstream targets of Foxo3a, between control and Sirt3-knock-
A

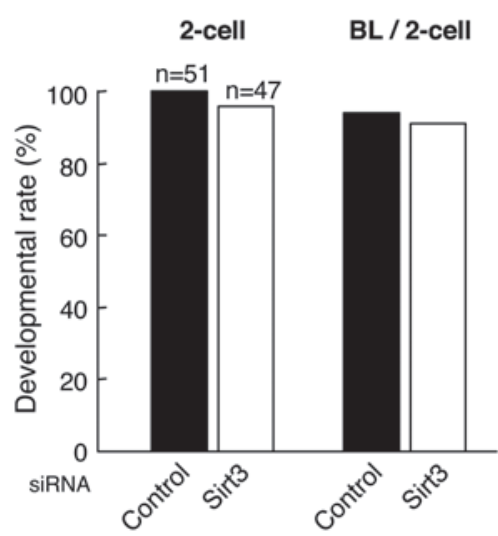

B

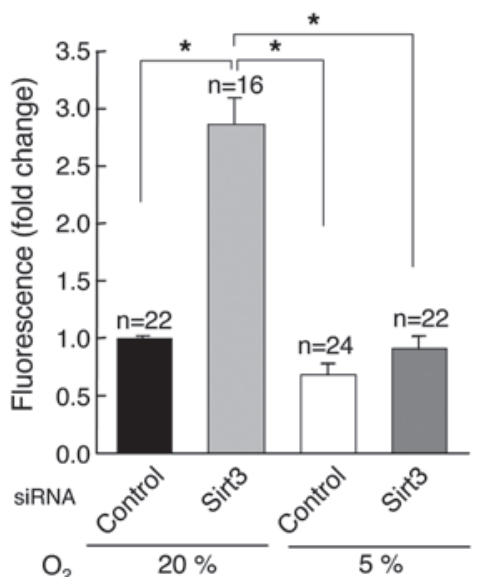

C

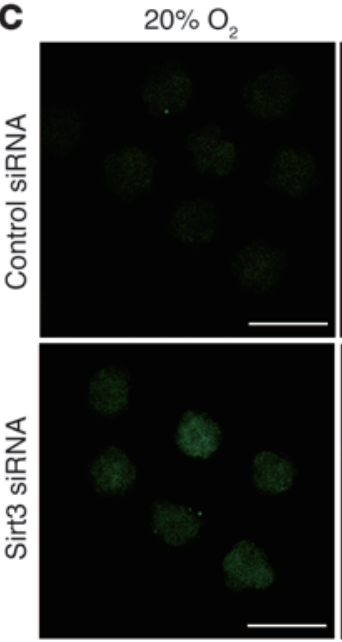

$5 \% \mathrm{O}_{2}$

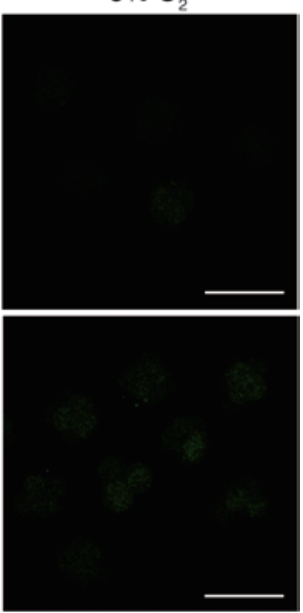

Figure 7

Low-oxygen conditions abolish developmental defects induced by Sirt3 knockdown. (A) Intracytoplasmic injection of Sirt3 siRNA did not affect 2-cell and blastocyst formation rates in low-oxygen $\left(5 \% \mathrm{O}_{2}\right)$ conditions. Data are derived from 4 independent experiments. (B) Effects of siRNA-mediated Sirt3 knockdown on intracellular ROS levels, as estimated by $\mathrm{CM}-\mathrm{H}_{2}$ DCFDA fluorescence intensity, in $20 \%$ and $5 \% \mathrm{O}_{2}$ conditions. Sirt3 knockdown-induced ROS increases were canceled in $5 \% \mathrm{O}_{2}$ conditions. Data are derived from 3 independent experiments. Statistical assessments were performed by applying Games-Howell test. ${ }^{*} P<0.05$. (C) Representative images of $\mathrm{CM}-\mathrm{H}_{2} \mathrm{DCFDA}$ fluorescence in embryos analyzed in $\mathbf{B}$. Scale bar: $100 \mu \mathrm{m}$. 


\section{Table 1}

Effect of maternal Sirt3 genotype on IVF rate

\begin{tabular}{lcccc}
\multicolumn{2}{l}{ Genotype } & \multicolumn{2}{c}{ IVF $(\boldsymbol{n})$} & Fertilization rate \\
Sperm & Egg & Fertilized & Unfertilized & \\
Sirt3 $^{+}$ & Sirt3 $^{+}$ & 51 & 19 & $73 \%$ \\
Sirt3 $^{+}$ & Sirt3 $^{-}$ & 62 & 28 & $69 \%$ \\
Sirt3- $^{-}$ & Sirt3 $^{+}$ & 62 & 10 & $86 \%$ \\
Sirt3- $^{-}$ & Sirt3- $^{-}$ & 65 & 31 & $68 \%$ \\
\hline
\end{tabular}

Cochran-Mantel-Haenszel $\chi^{2}$ test stratified on sperm genotype.

$\chi^{2}=4.701 ; P=0.030$; odds ratio 1.813 .

down embryos (our unpublished observations), which indicates that Foxo3a might not be contributing to the protective role of Sirt3 against oxidative stress in preimplantation embryos.

Involvement of p53 in ROS-induced preimplantation developmental arrest in Sirt3-deficient embryos. Mitochondrial ROS increase in Sirt3-knockdown embryos is accompanied by upregulation of p53 expression. Furthermore, siRNA-induced p53 knockdown improved developmental outcome in Sirt3-knockdown embryos.

Under normal conditions, levels of p53 protein are kept low because of its short half-life through the ubiquitin-proteasome degradation pathway, mediated by E3 ubiquitin ligase $\mathrm{Mdm} 2$, in preimplantation embryos as well as in various somatic cells (35, 46). Upon stress conditions, modification (e.g., phosphorylation) of $\mathrm{p} 53$ and/or $\mathrm{Mdm} 2$ causes $\mathrm{p} 53$ stabilization and activation by inhibiting the p53-Mdm2 interaction (35). In in vitro-cultured preimplantation embryos, the latency of p53 is maintained by the phosphatidylinositol-3'-kinase/Akt-mediated activation of Mdm2 (46). Perturbation of this pathway, as well as Mdm2 inactivation, results in the accumulation of p53 and developmental arrest (46-48), which indicates that the latency of p53 is important for normal preimplantation development. Although mitochondrial ROS has proved to contribute to stress-induced p53 activation in some somatic cells (49), the involvement of p53 in the stress response of preimplantation embryos has been controversial $(50,51)$. The present study indicated that p53 is involved in developmental arrest induced by mitochondrial ROS in Sirt3-knockdown embryos.

Sirt3-related mechanisms independent of ROS may be involved in p53 activation in cooperation with mitochondrial ROS. AMPK, which responds to an increase in AMP/ATP ratio during calorie restriction, can activate $\mathrm{p} 53$ by increasing transcription of $p 53$ and through direct phosphorylation of p53 $(52,53)$. This pathway might be activated in Sirt3-deficient embryos, in which mitochondrial ATP synthesis is possibly decreased (30). Another possibility is that Sirt 3 may deacetylate p53 as well as $\operatorname{Sirt} 1(54,55)$, and, in the absence of Sirt3, acetylated p53 may accumulate in embryos. p53 acetylation is induced in response to stress and causes its activation and stabilization (54-56). Furthermore, p53 has recently been reported to localize to mitochondria (57). Thus, it would be interesting to test whether acetylated p53 could serve as a substrate for Sirt3 deacetylase activity.

The present study showed that expression of Nanog was downregulated in Sirt3-knockdown or -knockout embryos, and expression of $p 21$ was upregulated. Although it may simply reflect developmental delay, this finding is compatible with previous reports that p53 suppresses Nanog expression in ES cells after DNA damage, which may serve as a mechanism to maintain genomic stability in ES cells $(39,40)$. We speculate that, in the absence of Sirt3, p53 is activated by excessive mitochondrial ROS to prevent the generation of embryos carrying serious mutations partly through changes in p21 and Nanog activity.

Conclusion. We have identified Sirt 3 as a protective factor against stress in preimplantation development under IVF and in vitro culture conditions. Sirt 3 deficiency caused increased mitochondrial ROS production and subsequent developmental arrest attributed to $\mathrm{p} 53$ activation in preimplantation embryos. The present findings may contribute to the understanding of preimplantation biology and give a clue to the better outcome of assisted reproductive technologies.

\section{Methods}

Mice. ICR mice from Charles River Laboratories Japan Inc. were used in all experiments, except for those using C57BL6-background Sirt3-knockout mice with the Sirt3 ${ }^{\text {Gt }(\text { neo) } 218 L e x}$ allele (here referred to as Sirt3-), which were generated by Lexicon Genetics and obtained from Mutant Mouse Regional Resource Centers. Mice were housed in an environmentally controlled room at $23^{\circ} \mathrm{C} \pm 2{ }^{\circ} \mathrm{C}$, with $50 \%-60 \%$ relative humidity, under a 12 -hour light/12-hour dark cycle. All animal experiments were approved by the Animal Care and Use Committee of the University of Tokyo and were performed in accordance with institutional guidelines.

Collection of eggs, IVF, and assessment of in vitro development. Female mice (8-10 weeks old) were superovulated with intraperitoneal injections of 7.5 IU pregnant mare serum gonadotropin (PMSG; Teikoku Hormone Mfg.) and, 48 hours later, 7.5 IU human chorionic gonadotropin (hCG; Teikoku Hormone Mfg.). Eggs were recovered from the oviducts 20 hours after hCG injection, and cumulus cells were extensively dispersed with $1 \mathrm{mg} / \mathrm{ml}$ hyaluronidase (Sigma-Aldrich). Sperm were expelled from the cauda epididymis of male mice into $200 \mu \mathrm{l}$ human tubal fluid (HTF) medium and incubated under mineral oil for $1-2$ hours at $37^{\circ} \mathrm{C}$ to capacitate. A sperm suspension at a concentration of $6-7 \times 10^{5} \mathrm{sperm} / \mathrm{ml}$ was used to inseminate eggs in a $200-\mu \mathrm{l}$ drop of HTF medium under mineral oil. After coincubation with sperm for 6 hours, the inseminated eggs were washed and cultured in 50- $\mu 1$ droplets of potassium simplex optimized medium (KSOM; Specialty Media) under mineral oil at $37^{\circ} \mathrm{C}$ in humidified air containing $5 \% \mathrm{CO}_{2}$. Experiments at low oxygen concentration were performed in a humidified airtight chamber maintained at $37^{\circ} \mathrm{C}$ and flushed with a mixture of $5 \% \mathrm{O}_{2}$ and $5 \% \mathrm{CO}_{2}$ balanced with $\mathrm{N}_{2}$. In experiments with inhibitors, each medium was supplemented with $5 \mathrm{mM}$ nicotinamide (Sigma-Aldrich), $200 \mu \mathrm{M}$ sirtinol (Calbiochem), $2 \mu \mathrm{M}$ BML-210 (Biomol), $500 \mu \mathrm{M}$ NAC (Wako), or DMSO (Sigma-Aldrich) alone. Cleavage and embryo development were examined every 24 hours.

\section{Table 2}

Effect of maternal Sirt3 genotype on blastocyst formation rate after IVF

\begin{tabular}{lcccc}
\multicolumn{2}{l}{ Genotype } & \multicolumn{2}{c}{ Blastocysts $(\boldsymbol{n})$} & Formation rate \\
Sperm & Egg & Formed & Unformed & \\
Sirt3 $^{+}$ & Sirt3 $^{+}$ & 30 & 1 & $97 \%$ \\
Sirt3 $^{+}$ & Sirt3 $^{-}$ & 19 & 13 & $59 \%$ \\
Sirt3- $^{-}$ & Sirt3 $^{+}$ & 39 & 3 & $93 \%$ \\
Sirt3- $^{-}$ & Sirt3- $^{-}$ & 30 & 15 & $67 \%$ \\
\hline
\end{tabular}

Cochran-Mantel-Haenszel $\chi^{2}$ test stratified on sperm genotype. $\chi^{2}=19.263 ; P<0.001$; odds ratio 9.666 . 

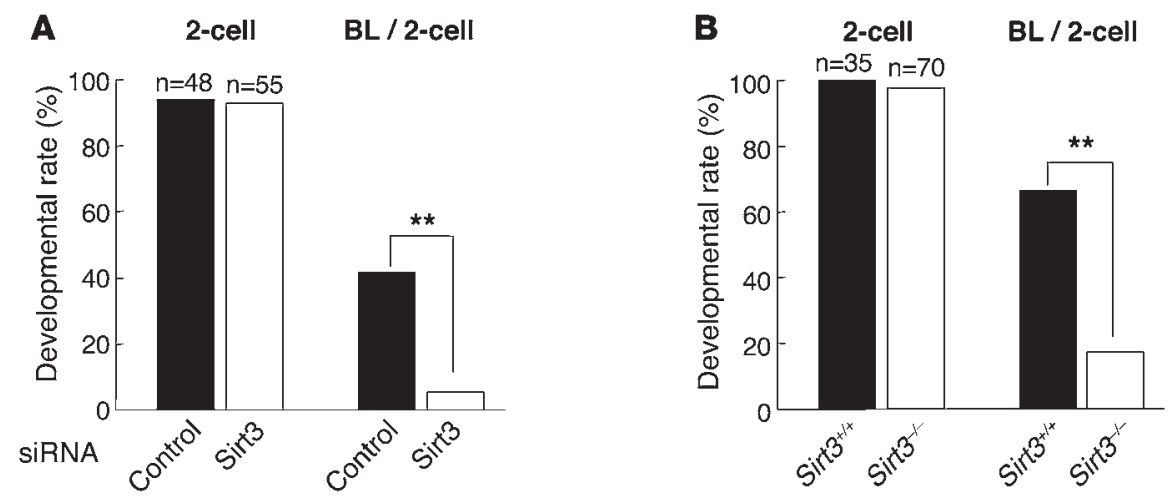

\section{Figure 8}

Parthenogenetic development is blocked by Sirt3 deficiency. (A) After $\mathrm{Sr}^{2+}$ activation for 4.5 hours, eggs that formed 2 pronuclei were injected with control or Sirt3 siRNA, and the rates of 2-cell and blastocyst formation were evaluated. (B) Eggs collected from wild-type and Sirt3-1- female mice underwent parthenogenetic activation. Data are derived from 4 (A) or 2 (B) independent experiments. Statistical assessments were performed by applying Fisher's exact test. ${ }^{* *} P<0.001$.

Assessment of in utero development. Female ICR mice (8-10 weeks old) were bred with vasectomized ICR males to stimulate pseudopregnancy. Vaginal plug-positive females were used as recipients. Cultured embryos were transferred to recipients on day 0.5 of pseudopregnancy according to standard procedures. 7-10 embryos were transferred into each fallopian tube. Recipients were subjected to cesarean section on day 18.5 to determine the developmental competence of transferred embryos.

Parthenogenetic activation of eggs. ICR mouse eggs were activated by a 3- to 4-hour treatment with $10 \mathrm{mM} \mathrm{Sr}^{2+}$ prepared in $\mathrm{Ca}^{2+}$-free M16 medium. Cytochalasin B (Sigma-Aldrich), an inhibitor of actin filament polymerization, was used to suppress the second polar body extrusion to generate diploid parthenotes. Activated eggs with 2 pronuclei, visualized under a differential interference contrast microscope, were defined as diploid parthenotes. Activated eggs were washed extensively and cultured in vitro under the same conditions as were IVF zygotes.

Plasmids. Mouse Sirt1, Sirt2, and Sirt3 cDNAs were cloned by RT-PCR on total RNA from NIH 3T3 cells. For EGFP fusion constructs, cDNA fragments encompassing the open reading frame of Sirt1, Sirt2, and Sirt3 were subcloned in frame into the PEGFP-N3 expression vector
(Clontech). The fragments encoding the fusion constructs were then subcloned into pCRII-TOPO (Invitrogen) for in vitro transcription. The constructs were verified by sequencing.

Cell culture and transfection. NIH 3T3 cells were cultured in Dulbecco modified Eagle medium containing $10 \%$ fetal calf serum and antibiotics at $37^{\circ} \mathrm{C}$ in $5 \% \mathrm{CO}_{2}$. For siRNA transfection, cells were grown to $30 \%-50 \%$ confluence and treated with a mixture of siRNA and oligofectamine reagents (Invitrogen) according to the manufacturer's protocol.

Conventional RT-PCR. Female ICR mice (8-10 weeks old) were superovulated as above and used for collection of eggs or mated with ICR male mice to obtain early embryos at different stages. In some experiments, in vitro-cultured embryos and NIH 3 T3 cells were collected at the indicated times. Samples were lysed in lysing buffer (Isogen; Nippon Gene), with volumes adjusted according to the number of eggs or embryos, and subjected to RNA extraction according to standard procedures. After reverse transcription with Quantiscript Reverse Transcriptase and RT Primer Mix (QuantiTect Reverse Transcription; Qiagen), PCR amplification was performed using specific primers listed in Supplemental Table 1 . Thermal cycling was performed for 30-35 cycles
A

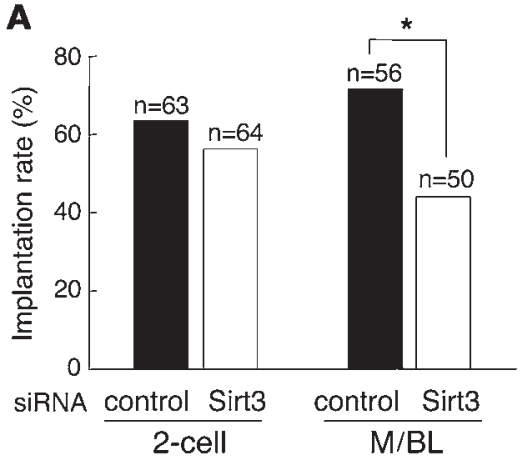

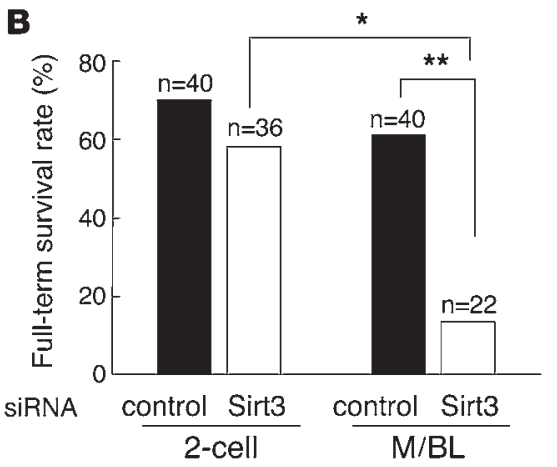

Figure 9

Long-term in vitro culture leads to decreased implantation and full-term survival rates in Sirt3-knockdown embryos. (A and B) Implantation rates (A) and full-term survival rates (B) of embryos injected with Sirt3 or control siRNA and transferred into pseudopregnant mice at the 2-cell or morula/blastocyst stage. Implantation sites and viability of fetuses were inspected by cesarean section 18 days after transfer. Implantation rate was estimated by the number of implantation sites; full-term survival rate was assessed by dividing the number of live pups by the number of implantation sites. Data are derived from 5 (2-cell embryo transfer) or 4 (morula/blastocyst transfer) independent experiments. Statistical assessments were performed by applying Ryan's multiple-comparison test. ${ }^{*} P<0.005$; ${ }^{*} P<0.001$. 
A

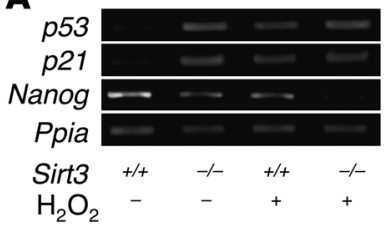

C

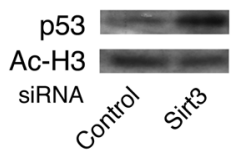

B

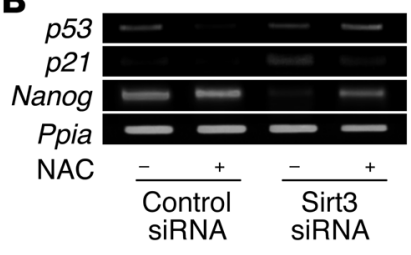

\section{Figure 10}

Involvement of p53 in developmental arrest of Sirt3-deficient preimplantation embryos. (A) p53 and p21 were upregulated in Sirt3-embryos as in $\mathrm{H}_{2} \mathrm{O}_{2}$-treated wild-type embryos. Nanog expression was decreased in Sirt3 ${ }^{-/-}$embryos, and the decrease was enhanced by $\mathrm{H}_{2} \mathrm{O}_{2}$ stimulus. (B) Effects of Sirt3 knockdown and treatment with NAC on the expression of $p 53$ and its downstream genes. In Sirt3 siRNAinjected embryos, p21 expression was upregulated, whereas Nanog expression was downregulated. These effects were blocked by NAC. (C) Western blotting analysis showing increased p53 protein levels in Sirt3-knockdown embryos at the morula stage. Signals for acetylated histone $\mathrm{H} 3(\mathrm{Ac}-\mathrm{H} 3)$ served as an internal control. (D) Effects of p53 knockdown on Sirt3 siRNA-induced changes in the expression of genes downstream of $\mathrm{p} 53$. Sirt3 siRNA-induced $p 21$ upregulation and Nanog downregulation were blocked by siRNA-mediated p53 knockdown. Ppia expression served as an internal control in A, B, and D. (E) Effects of p53 knockdown on preimplantation developmental arrest in Sirt3-knockdown embryos. The rate of blastocyst formation was significantly improved by coinjection with $\mathrm{p} 53$ siRNA. Data are derived from 4 independent experiments. Statistical assessments were performed by applying Ryan's multiple-comparison test. ${ }^{*} P<0.05$; ${ }^{* *} P<0.001$.
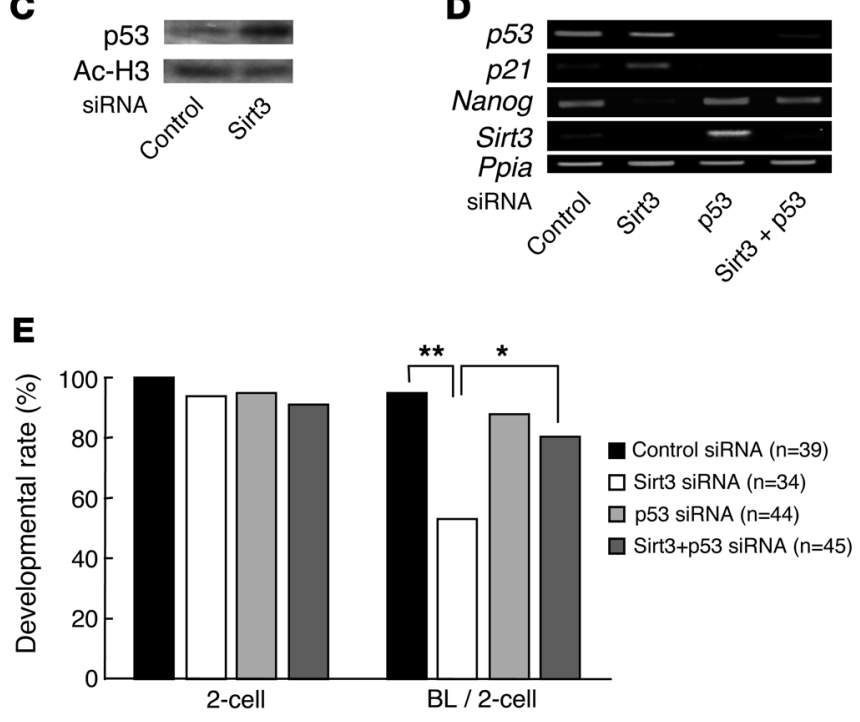

to maintain PCR conditions within the linear range of amplification before saturation was reached. Each cycle consisted of 30 seconds of denaturation at $94^{\circ} \mathrm{C}, 30$ seconds of annealing at each annealing temperature (Supplemental Table 1), and 30 seconds of extension at $72^{\circ} \mathrm{C}$. Signals for Gapdh and peptidylprolyl isomerase A (Ppia) expression served as internal controls.

Quantitative real-time PCR. Quantification of each mRNA level was performed by real-time RT-PCR analyses using a LightCycler (Roche) and Real-Time PCR Premix with SYBR Green (RBC Bioscience) following the manufacturers' protocols. Each cycle consisted of 10 seconds of denaturation at $94^{\circ} \mathrm{C}, 13-15$ seconds of annealing at each annealing temperature (Supplemental Table 2), and 12-13 seconds of extension at $72^{\circ} \mathrm{C}$. Thermal cycling was performed for $45-50$ cycles. The secondderivative maximum method was adopted to determine the crossing points automatically for individual samples, and relative amounts of mRNA were calculated based on the crossing-point analysis. Ppia was used as an internal control. The result was expressed as fold change relative to control.

Western blotting. A pool of approximately 50 early embryos or NIH 3T3 cells was lysed in Laemmli buffer containing protease inhibitors, and then subjected to $12 \%$ SDS-PAGE. The separated proteins were transferred to a nylon membrane, which was then pretreated with $3 \%$ bovine serum albumin for blocking and incubated with primary antibodies as follows: rabbit anti-Sirt3 antibody (Abgent) for NIH 3T3 cell samples, rabbit anti-Sirt3 antibody from E. Verdin (UCSF, San Francisco, California, USA; ref. 26) for early embryo samples, and mouse anti-p53 antibody (BD Biosciences - Pharmingen). Blots were then incubated with anti-rabbit or anti-mouse IgG antibody conjugated to horseradish peroxidase (ICN). The protein bands were visualized using an ECL Plus Western Blotting Detection System (Amersham Pharmacia Biotech). The membrane was then washed and reblotted with anti- $\alpha$-tubulin

antibody (Sigma-Aldrich) or anti-acetylated histone H3 antibody (Cell Signaling Technology) for an internal control. Densitometric quantification was performed with ImageJ software (NIH).

RNAi. Chemically synthesized 25-nucleotide stealth RNAi duplex oligonucleotides were commercially obtained (Invitrogen). We selected 2 different sequences for each of the genes, Sirt 3 and $p 53$, selected for the generation of siRNA: the sequences for siRNAs 1 and 2 corresponded, respectively, to nucleotides 355-379 and 386-410 of mouse Sirt3 (GenBank accession no. NM_022433) and to nucleotides $985-1,009$ and $165-189$ of mouse $p 53$ (GenBank accession no. NM_011640; Supplemental Table 2). Stealth RNAi negative control HiGC (Invitrogen) was used as control siRNA. Approximately $10 \mathrm{pl}$ of $20 \mu \mathrm{M}$ siRNA was injected into the cytoplasm of fertilized eggs. The efficiency of gene knockdown was evaluated by RT-PCR with primers specific for mouse Sirt3 and p53 mRNA (Supplemental Table 1).

In vitro transcription and RNA microinjection. Preparation and injection of in vitro-transcribed mRNA were performed as previously described (58). Briefly, plasmids containing Sirt1-EGFP, Sirt2-EGFP, and Sirt3-EGFP fusion constructs and EGFP alone were linearized and used as templates for in vitro transcription using the T7 MessageMachine kit (Ambion). Synthesized RNA was further polyadenylated by yeast poly(A) polymerase (Amersham Biosciences) and resolved in $150 \mathrm{mM} \mathrm{KCl}$ with a final concentration of approximately $100 \mathrm{ng} / \mu \mathrm{l}$. The diluted RNA was filtered, heated at $90^{\circ} \mathrm{C}$ for 1 minute, and cooled on ice. Then, approximately $10 \mathrm{pl} \mathrm{RNA}$ solution was injected into fertilized eggs through a glass micropipette. Embryos were grown to the 2-cell stage, at which time confocal images were obtained using a Nikon D-ECLIPSE C1 system.

Detection of intracellular ROS. To detect intercellular ROS in living embryos, we used $\mathrm{CM}-\mathrm{H}_{2}$ DCFDA from Invitrogen. $\mathrm{CM}-\mathrm{H}_{2}$ DCFDA was prepared in DMSO immediately prior to loading. Embryos were incubated with $10 \mu \mathrm{M}$ CM- $\mathrm{H}_{2}$ DCFDA for 30 minutes and observed under a laser scanning confocal microscope (Nikon D-ECLIPSE C1), with an excitation wavelength of 
$480 \mathrm{~nm}$ and an emission wavelength of 505-530 nm. In some experiments, embryos were pretreated with the $\mathrm{NAD}(\mathrm{P}) \mathrm{H}$ oxidase inhibitor apocynin $(100 \mu \mathrm{M})$ or the ubiquinol/cytochrome $c$ oxidoreductase inhibitor stigmatellin $(10 \mu \mathrm{M})$ for 30 minutes.

Statistics. Differences between 2 groups were analyzed by Mann-Whitney $U$ test. Multiple comparisons between more than 2 groups were analyzed by 1-way ANOVA and post-hoc tests as indicated in the figure legends. Continuous data are presented as mean \pm SEM. $P$ values less than 0.05 were considered significant.

\section{Acknowledgments}

We thank Eric Verdin for Sirt3 antibody; Atsuo Ogura, Hiroshi Suzuki, and Kiyoshi Kita for helpful discussions and comments; Yuko Fujisawa for technical assistance; and Chisato Fujimoto for statistical analysis. This work was supported in part by Global COE Program (Integrative Life Science Based on the Study of Biosignaling Mechanisms); MEXT, Japan; grants-in-aid for scientific research from the Ministry of Education, Culture, Sports, Science and Technology of Japan; and grants-in-aid for scientific research from the Ministry of Health, Labor, and Welfare of Japan.

Received for publication December 14, 2009, and accepted in revised form June 9, 2010.

Address correspondence to: Hiroki Kurihara, Department of Physiological Chemistry and Metabolism, Graduate School of Medicine, University of Tokyo, 7-3-1 Hongo, Bunkyo-ku, Tokyo 113-0033, Japan. Phone: 81.3.5841.3498; Fax: 81.3.5684.4958; E-mail: kuri-tky@umin.ac.jp.

Nanao Horike's present address is: Animal Models of Human Diseases, National Institute of Biomedical Innovation, Osaka, Japan.

Tomokazu Amano's present address is: Developmental Genomics and Aging Section, Laboratory of Genetics, National Institute on Aging, NIH, Baltimore, Maryland, USA.
1. Matzuk MM, Lamb DJ. The biology of infertility: research advances and clinical challenges. Nat Med. 2008;14(11):1197-1213.

2. Smith S, Pfeifer SM, Collins JA. Diagnosis and management of female infertility. JAMA. 2003; 290(13):1767-1770.

3. Van Voorhis BJ. Clinical practice. In vitro fertilization. N Engl J Med. 2007;356(4):379-386.

4. Baird DD, Wilcox AJ, Weinberg CR. Use of time to pregnancy to study environmental exposures. AmJ Epidemiol. 1986;124(3):470-480.

5. Witschi E. Natural control of fertility. Fertil Steril. 1968;19(1):1-14

6. Van Blerkom J. Mitochondria in early mammalian development. Semin Cell Dev Biol. 2009; 20(3):354-364.

7. Wilding M, Coppola G, Dale B, Di Matteo L. Mitochondria and human preimplantation embryo development. Reproduction. 2009;137(4):619-624.

8. Thompson JG, McNaughton C, Gasparrini B, McGowan LT, Tervit HR. Effect of inhibitors and uncouplers of oxidative phosphorylation during compaction and blastulation of bovine embryos cultured in vitro. J Reprod Fertil. 2000;118(1):47-55.

9. Thouas GA, Trounson AO, Wolvetang EJ, Jones GM. Mitochondrial dysfunction in mouse oocytes results in preimplantation embryo arrest in vitro. Biol Reprod. 2004;71(6):1936-1942.

10. Trimarchi JR, Liu L, Porterfield DM, Smith PJ, Keefe DL. Oxidative phosphorylation-dependent and -independent oxygen consumption by individual preimplantation mouse embryos. Biol Reprod. 2000;62(6):1866-1874.

11. Van Blerkom J, Davis PW, Lee J. ATP content of human oocytes and developmental potential and outcome after in-vitro fertilization and embryo transfer. Hum Reprod. 1995;10(2):415-424.

12. Raha S, Robinson BH. Mitochondria, oxygen free radicals, disease and ageing. Trends Biochem Sci. 2000;25(10):502-508.

13. Zhang DX, Gutterman DD. Mitochondrial reactive oxygen species-mediated signaling in endothelial cells. Am J Physiol Heart Circ Physiol. 2007; 292(5):H2023-H2031.

14. Brookes PS, Yoon Y, Robotham JL, Anders MW, Sheu SS. Calcium, ATP, and ROS: a mitochondrial love-hate triangle. Am J Physiol Cell Physiol. 2004;287(4):C817-C833.

15. Covarrubias L, Hernandez-Garcia D, Schnabel D, Salas-Vidal E, Castro-Obregon S. Function of reactive oxygen species during animal development: passive or active? Dev Biol. 2008;320(1):1-11.

16. Ruder EH, Hartman TJ, Blumberg J, Goldman MB. Oxidative stress and antioxidants: exposure and impact on female fertility. Hum Reprod Update. 2008;14(4):345-357.

17. Agarwal A, Gupta S, Sekhon L, Shah R. Redox considerations in female reproductive function and assisted reproduction: from molecular mechanisms to health implications. Antioxid Redox Signal. 2008;10(8):1375-1403.

18. Liu L, Trimarchi JR, Keefe DL. Involvement of mitochondria in oxidative stress-induced cell death in mouse zygotes. Biol Reprod. 2000;62(6):1745-1753.

19. Finkel T, Deng CX, Mostoslavsky R. Recent progress in the biology and physiology of sirtuins. Nature. 2009;460(7255):587-591.

20. Schwer B, Verdin E. Conserved metabolic regulatory functions of sirtuins. Cell Metab. 2008;7(2):104-112.

21. Longo VD, Kennedy BK. Sirtuins in aging and agerelated disease. Cell. 2006;126(2):257-268.

22. Imai S, Armstrong CM, Kaeberlein M, Guarente L. Transcriptional silencing and longevity protein Sir2 is an NAD-dependent histone deacetylase. Nature. 2000;403(6771):795-800.

23. Revollo JR, Grimm AA, Imai S. The NAD biosynthesis pathway mediated by nicotinamide phosphoribosyltransferase regulates Sir 2 activity in mammalian cells. J Biol Chem. 2004;279(49):50754-50763.

24. Onyango P, Celic I, McCaffery JM, Boeke JD, Feinberg AP. SIRT3, a human SIR2 homologue, is an NADdependent deacetylase localized to mitochondria. Proc Natl Acad Sci US A. 2002;99(21):13653-13658.

25. Scher MB, Vaquero A, Reinberg D. SirT3 is a nuclear NAD+-dependent histone deacetylase that translocates to the mitochondria upon cellular stress. Genes Dev. 2007;21(8):920-928.

26. Schwer B, North BJ, Frye RA, Ott M, Verdin E. The human silent information regulator (Sir)2 homologue hSIRT3 is a mitochondrial nicotinamide adenine dinucleotide-dependent deacetylase. J Cell Biol. 2002;158(4):647-657.

27. Shi T, Wang F, Stieren E, Tong Q. SIRT3, a mitochondrial sirtuin deacetylase, regulates mitochondrial function and thermogenesis in brown adipocytes. J Biol Chem. 2005;280(14):13560-13567.

28. Hallows WC, Lee S, Denu JM. Sirtuins deacetylate and activate mammalian acetyl-CoA synthetases. Proc Natl Acad Sci U S A. 2006;103(27):10230-10235.

29. Schwer B, Bunkenborg J, Verdin RO, Andersen JS, Verdin E. Reversible lysine acetylation controls the activity of the mitochondrial enzyme acetyl-CoA synthetase 2. Proc Natl Acad Sci U S A. 2006;103(27):10224-10229.

30. Ahn BH, et al. A role for the mitochondrial deacetylase Sirt3 in regulating energy homeostasis. Proc Natl Acad Sci U S A. 2008;105(38):14447-14452.

31. Lombard DB, et al. Mammalian Sir2 homolog
SIRT3 regulates global mitochondrial lysine acetylation. Mol Cell Biol. 2007;27(24):8807-8814.

32. Tsai FC, Gardner DK. Nicotinamide, a component of complex culture media, inhibits mouse embryo development in vitro and reduces subsequent developmental potential after transfer. Fertil Steril. 1994;61(2):376-382.

33. Fato R, et al. Differential effects of mitochondrial Complex I inhibitors on production of reactive oxygen species. Biochim Biophys Acta. 2009; 1787(5):384-392.

34. Dumollard R, Duchen M, Carroll J. The role of mitochondrial function in the oocyte and embryo. Curr Top Dev Biol. 2007;77:21-49.

35. Kruse JP, Gu W. Modes of p53 regulation. Cell. 2009;137(4):609-622.

36. Vousden KH, Ryan KM. p53 and metabolism. Nat Rev Cancer. 2009;9(10):691-700.

37. Menendez D, Inga A, Resnick MA. The expanding universe of p53 targets. Nat Rev Cancer. 2009;9(10):724-737.

38. Riley T, Sontag E, Chen P, Levine A. Transcriptional control of human p53-regulated genes. Nat Rev Mol Cell Biol. 2008;9(5):402-412.

39. Han MK, Song EK, Guo Y, Ou X, Mantel C, Broxmeyer HE. SIRT1 regulates apoptosis and Nanog expression in mouse embryonic stem cells by controlling p53 subcellular localization. Cell Stem Cell. 2008;2(3):241-251.

40. Lin T, et al. p53 induces differentiation of mouse embryonic stem cells by suppressing Nanog expression. Nat Cell Biol. 2005;7(2):165-171.

41. Sundaresan NR, Samant SA, Pillai VB, Rajamohan SB, Gupta MP. SIRT3 is a stress-responsive deacetylase in cardiomyocytes that protects cells from stress-mediated cell death by deacetylation of Ku70. Mol Cell Biol. 2008;28(20):6384-6401.

42. Wallace DC, Fan W. The pathophysiology of mitochondrial disease as modeled in the mouse. Genes Dev. 2009;23(15):1714-1736.

43. Jacobs KM, et al. SIRT3 interacts with the daf-16 homolog FOXO3a in the mitochondria, as well as increases FOXO3a dependent gene expression. Int J Biol Sci. 2008;4(5):291-299.

44. Turrens JF. Mitochondrial formation of reactive oxygen species. J Physiol. 2003;552(pt 2):335-344.

45. Sundaresan NR, Gupta M, Kim G, Rajamohan SB, Isbatan A, Gupta MP. Sirt3 blocks the cardiac hypertrophic response by augmenting Foxo3adependent antioxidant defense mechanisms in mice. J Clin Invest. 2009;119(9):2758-2771.

46. Jin XL, Chandrakanthan V, Morgan HD, O’Neill C. Preimplantation embryo development in the mouse requires the latency of TRP53 expression, 
which is induced by a ligand-activated PI3 kinase/ AKT/MDM2-mediated signaling pathway. Biol Reprod. 2009;81(1):234-242.

47. Jones SN, Roe AE, Donehower LA, Bradley A. Rescue of embryonic lethality in Mdm2-deficient mice by absence of p53. Nature. 1995;378(6553):206-208.

48. Montes de Oca Luna R, Wagner DS, Lozano G. Rescue of early embryonic lethality in $\mathrm{mdm} 2$ deficient mice by deletion of p53. Nature. 1995; 378(6553):203-206.

49. Karawajew L, Rhein P, Czerwony G, Ludwig WD. Stress-induced activation of the p53 tumor suppressor in leukemia cells and normal lymphocytes requires mitochondrial activity and reactive oxygen species. Blood. 2005;105(12):4767-4775.

50. Favetta LA, Robert C, St John EJ, Betts DH, King
WA. p66shc, but not p53, is involved in early arrest of in vitro-produced bovine embryos. Mol Hum Reprod. 2004;10(6):383-392.

51. Velez-Pardo C, Morales AT, Del Rio MJ, Olivera-Angel M. Endogenously generated hydrogen peroxide induces apoptosis via mitochondrial damage independent of NF-kappaB and p53 activation in bovine embryos. Theriogenology. 2007;67(7):1285-1296.

52. Jones RG, et al. AMP-activated protein kinase induces a p53-dependent metabolic checkpoint. Mol Cell. 2005;18(3):283-293.

53. Okoshi R, et al. Activation of AMP-activated protein kinase induces p53-dependent apoptotic cell death in response to energetic stress. J Biol Chem. 2008;283(7):3979-3987.

54. Luo J, et al. Negative control of p53 by Sir2alpha promotes cell survival under stress. Cell. 2001; 107(2):137-148.

55. Vaziri $\mathrm{H}$, et al. hSIR2(SIRT1) functions as an NAD-dependent p53 deacetylase. Cell. 2001; 107(2):149-159.

56. Ito A, et al. p300/CBP-mediated p53 acetylation is commonly induced by p53-activating agents and inhibited by MDM2. EMBO J. 2001; 20(6):1331-1340.

57. Marchenko ND, Wolff S, Erster S, Becker K, Moll UM. Monoubiquitylation promotes mitochondrial p53 translocation. EMBO J. 2007;26(4):923-934.

58. Aida T, Oda S, Awaji T, Yoshida K, Miyazaki S. Expression of a green fluorescent protein variant in mouse oocytes by injection of RNA with an added long poly(A) tail. Mol Hum Reprod. 2001;7(11):1039-1046. 